\title{
Stuff we Should Not Be Doing: A Look at Better Ways to Determine the Appropriate Tests to Offer and How to Best Minimize Errors. Antibiotic Stewardship and Laboratory Stewardship, How can they Help
}

\author{
Robert L Sautter* \\ Principle of RL Sautter Consulting LLC, Lancaster, USA \\ *Corresponding author: Robert L Sautter, Principle of RL Sautter Consulting LLC, Lancaster, South Carolina, USA. \\ To Cite This Article: Robert L Sautter, Stuff we Should Not Be Doing: A Look at Better Ways to Determine the Appropriate Tests to Offer and \\ How to Best Minimize Errors. Antibiotic Stewardship and Laboratory Stewardship, How can they Help. 2020 - 8(1). AJBSR.MS.ID.001222. \\ DOI: 10.34297 /AJBSR.2020.08.001222.
}

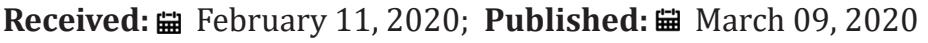

This commentary was previously published in MedLab Magazine; Sautter, Robert L. and Keven C. Hazen. Stuff we should not be Doing. A look at better ways to determine the appropriate tests to offer and also how to best minimize errors. 2/2019. https://www.medlabme.com/magazine/en/Issue/2018-issue-21/ stuff-we-should-not-be-doing.html Accessed 2/6/2020

\section{Editorial}

Laboratory testing is dependent upon Preanalytical, analytical and postanalytical test phases. In the laboratory we are focused upon the analytical phase and often ignore the preanalytical and postanalytical portion of testing. This is a drastic error, even though both the pre and post phases are more difficult to control, they account for many errors and for delays in caregivers reacting to test results. Seventy percent of laboratory errors are associated with pre-analytical errors. Much work has been done to speed the results of laboratory testing in the analytical phase, however if the results are not available to care givers soon after they are posted in the laboratory information system, then the effort expended to speed the testing will be wasted. When possible, electronic results sent directly to the care giver will aid in care, i.e. Theradoc system. Also, if test results can be in the medical record when the caregiver can review them or rounds with patients, it will speed appropriate care.

Over the years, we have often offered testing and methodologies that can be described as "we have always done it that way". The use of evidenced based medicine should replace that approach so that providers test the appropriate patient, use the best method to diagnose disease and treat patients appropriately. Microbiologists, infectious disease physicians and pharmacists have recently been engaged in antibiotic stewardship programs to better use appropriate antimicrobials to treat patients and not to use them on patients that do not need them. By doing so, a significant cost savings will be well as better outcomes for patients. Too often laboratory medical and administrative staff make decisions in a void without using expertise from other disciplines. The use of multidisciplinary teams and diagnostic management teams offer a great advantage in treating patients and offering relevant laboratory testing.

In addition to some practices mentioned above, during the "live" presentation some of the "stuff we should not be doing" is also detailed below.

Examples:

a. Accepting samples that are not appropriate for testing either due to poor collection, storage, or from unapproved sources without proper validation studies.

b. Adding newer methodologies without addressing with affected medical professionals preanalytical specimen collection errors and other pre-analytical steps.

c. Shipping samples many hours to the main laboratory if processing, testing or screening can be done locally. If they cannot be done in the laboratory, then in the Point of Care [1].

d. Adding new methodologies or procedures without discussing them with a multidisciplinary team including: Infectious disease, the physician specialty affected by the change and administration (i.e., the post-analytical impact of a new test). 
e. Agreeing to perform AST on drug-bug combinations for which there are no standards.

f. Other "do not do" items were discussed.

\section{Antibiotic Stewardship and the Laboratory}

The laboratory has played a key role in dispensing antimicrobial results as well as interpretation for clinicians, nurses and pharmacists. The explosion of antibiotic stewardship has occurred in the last few years with increasing full-time equivalents (FTE) as well as increasing budgets in pharmacy, infectious disease and nursing. However, since the Joint Commission issued a new standard for antimicrobial stewardship programs in hospitals, critical access hospitals, and nursing care centers [2], the laboratory has been excluded from the "table". The members of the team as mentioned in the standard are an infectious disease physician, pharmacist, practitioners and an infection preventionist. For several of the members of the team, "if available in the setting," are to be included. The laboratory and in particular the microbiology laboratory are excluded from the required active members. Ideally a doctoral level microbiologist should be on the team as is recognized by the CDC [3] Although the Joint Commission states that they mimic the requirements for the team published by $\mathrm{CDC}$, however, a member of microbiology laboratory or the diagnostic laboratory in general is not mentioned as key members of their stewardship team.

\section{Laboratory Stewardship}

Over the last 30 years an explosion in the cost of health care has occurred, the laboratory is not exempt to this issue. In fact, an estimated 4-5 billion laboratory tests are performed annually with $30 \%$ of them unnecessary [4]. The clinical microbiology laboratory is to provide appropriate tests to aid in diagnosis and therapeutic management of a patient with an infectious disease, evidence-based approaches are key to this success as well as using a multidisciplinary team and also diagnostic management teams to treat patients. The clinical microbiologist should not only be dispensing test results but also should be key in determining which tests are useful in a patient scenario along with other care givers. Many of tests that could be ordered are not pertinent to the disease but rather simply just "ordered by habit". Stewardship programs rely on the clinical microbiology laboratory to direct actions needed for effective stewardship activities.

Examples of those contributions to stewardship are:

a) Preanalytical, analytical and postanalytical test phases all influence the value of a test result

b) Rapid result reporting can be an extremely important parameter for care as it can lead to effective interventions

c) Guidance in selection of tests and interpretive assistance of the results d) Implementation of the most currently effective testing modalities and interpretation of the results; reporting appropriate antibiotics associated with positive outcomes for the patient

e) Detection of antibiotic resistance begins in the Clinical Microbiology laboratory.

By working with other health care specialties, the microbiologist can serve as a valuable resource to the health care system. There are many other examples of how the microbiologist contributes to antibiotic stewardship, some of which may be found in Sautter \& Halstead [4-6].

The clinical microbiologist plays an important role in antibiotic stewardship and contributes to the antibiotic and laboratory stewardship teams in many ways, some of which are detailed above. They should be part of hospital, state, regional and federal expert panels to come up with guidelines on all areas of microbiology, as demonstrated in North Carolina's CRE guidance [7]. To that point, clinical microbiology should be represented on all committees discussing antibiotic resistance and stewardship and infection control so the clinical microbiologist can make clear how he/she can help.

At MedLab Middle East, I (RLS) gave a presentation 0n "Stuff we should not be doing" and expanded upon how the microbiologist should approach these issues.

\section{References}

1. Sautter Robert L, Dawn M Earnest, DC Halstead (2018) What's Old Is New Again: Laboratory Oversight of Point of Care Testing-Guidelines, Challenges, and Practical Strategies, Clin Micro News: accepted for publication $40(23)$.

2. (2016) The Joint Commission. Approved: new antimicrobial stewardship standard. Joint Commission Perspectives 36: 1-8.

3. (2017) Centers for Disease Control and Prevention. Posting date. Core elements of hospital antibiotic stewardship programs. Centers for Disease Control and Prevention.

4. Halstead DC, Sautter RL (2018) New paradigm, new opportunities: laboratory stewardship, Clin Micro News 40(21): 175-180.

5. Sautter RL, Halstead DC (2018) Need of the hour: addressing the challenges of multi-drug-resistant health care-associated infections and the role of the laboratory in lowering infection rates. Clin Microbiol Newsletter 40: 11-16.

6. Barlam TF, Cosgrove SE, Abbo LM, MacDougall C, Schuetz AN, Septimus $\mathrm{E}$, et al. (2016) Implementing an antibiotic stewardship program: guidelines by the Infectious Diseases Society of America and the Society for Healthcare Epidemiology of America. Clinical infectious diseases: an official publication of the Infectious Diseases Society of America 62(10): e51-77.

7. (2018) Carbapenem-Resistant Enterobacteriaceae (CRE) Screening and Confirmatory Testing Recommendations in North Carolina 2018, North Carolina CRE Laboratory Task Force, Oct 1. 